none of Professor Davis' qualms about their identity. Their voice in the world of medical science and in the corridors of power in Washington is clear and strong. Our colleagues in Europe unashamedly identify paediatrics as a science and profession looking, with a particular eye to 1992, for counterpart cooperation from the highly respected and reputed body of paediatricians in this country. Our colleagues in more distant parts of the world turn to British paediatricians for leadership and support in their clinical and political struggles for the betterment of their children's health. The formation of an independent college for paediatricians and those involved in children's health work would, I believe, be universally welcomed.

I would like to agree with Professor Davis on one point. We should indeed look for and confidently follow the firm leadership of our elected officers whose discussion document on proposals for a college is currently in its final stages of preparation.

\section{Reflex anal dilatation associated with severe chronic constipation}

Sirs,

The flurry of publications in both the medical and the popular press about the significance, or otherwise, of reflex anal dilatation is serving only to confuse the issue further, and to compromise the position of careful and caring doctors who are doing their best to help abused children and their families.

The article by Clayden appears to imply that a visibly relaxed sphincter is synonymous with reflex anal dilatation. ${ }^{1}$ We know that a lax anal sphincter occurs in the absence of reflex anal dilatation. Is a lax sphincter the same as a visibly relaxed sphincter?

The children described in Clayden's series had all been subjected to invasive techniques of management, including suppositories, enemas, anal dilatation under anaesthesia, anoplasty and, in two children, internal anal sphincterotomy. It is therefore hardly surprising that $15 \%$ of these children showed 'a relaxed sphincter'. They were the subjects of trauma similar to that associated with anal abuse. Surely the lesson to be learnt from this finding is that it is unsafe to diagnose sexual abuse on the basis of anal dilatation in a child who is clearly grossly constipated, and who has been subjected to anal penetration. Reflex anal dilatation is a physical sign and like all physical signs has a differential diagnosis, in this instance including anal abuse.

It is time that some precision is brought into the terminology of reflex anal dilatation so that we can be certain that we are all talking about the same thing. From the present literature, it is often difficult to know whether authors are referring merely to relaxation of the external sphincter, to a lax sphincter, or to reflex anal dilatation. This type of misunderstanding may be the basis for the claim that reflex dilatation is a commonly seen and unimportant finding. ${ }^{2}$ Such a view seems to be favoured by doctors dealing mainly with adult patients. Few, if any, paediatricians claim to see gross reflex dilatation fre- quently, unless in anally abused children. Any description of reflex anal dilatation should include the actual measurement of the diameter of the open anal canal, together with a description of both the persistence, and the reproducibility of the sign. It is vital that such documentation is given so that the importance of the data can be assessed.

\section{References \\ ${ }^{1}$ Clayden GS. Reflex anal dilatation associated with severe chronic constipation. Arch Dis Child 1988;63:832-6. \\ 2 Heald RJ. Child abuse. Br Med J 1988;297:421.}

B L PRIESTLEY and L S TAITZ

The Children's Hospital, Sheffield S10 2TH

\section{Dr Clayden comments:}

Sadly this letter reflects the unhealthy attitude that criticism of scientific detail is identical to opposition to the whole movement to reduce child sexual abuse. The 'flurry of publications' is symptomatic of the previous dearth of evidence on the reliability of the anal signs of child sex abuse and of information about those conditions which might mimic these signs. My publications were an attempt to warn those careful and caring doctors not to compromise themselves by trusting to signs which may mislead them. ${ }^{12}$ Apart from the obvious trauma to the children and families, incorrect diagnosis of sex abuse sets back the whole momentum of vigilance and credibility in the population in general.

Either my lack of clarity or Priestley and Taitz's cognitive disonance has led to a degree of misunderstanding. They have read the surgical interventions given in table 5 as having occurred before presentation to my clinic whereas it describes the treatment given on diagnosis and was included to emphasise the severity of the chronic constipation in this group. All but three of the children with a visibly relaxed sphincter had had invasive anal treatment before presentation to my clinic (table 4), however, I know of recent cases where child sex abuse was confidently diagnosed on anal findings despite clear histories of invasive anal procedures.

Priestley and Taitz reasonably object to my use of the term 'visibly relaxed sphincter'. This was the term I had used at the beginning of this decade to collect the data and therefore it is accurate to describe it as such. In a small prospective study of the appearance of the anus in similarly chronically constipated children I found a number with a visibly lax sphincter who on occasions had reflex anal dilatation and on other occasions had a normal appearance. I agree that it is time for a proper definition based on accurate measurement of size and time.

As the rectoanal reflex is an essential part of the physiology of all but those with Hirschsprung's disease, the appearance of reflex anal dilatation is inevitable if the external sphincter is held apart (it fatigues in approximately 30 seconds in most subjects) and the rectum contains a stool which evokes the rectoanal reflex. Obviously if the external anal sphincter is damaged by frequent buggery this reflex is likely to be more apparent but so it will be if the child has not practised using the external sphincter. 
This may be because the anus has been partially obstructed or the child has not been trained as in early childhood and infancy or in severe developmental delay. Obviously neuromuscular conditions such as spina bifida or muscle disease will also appear abnormal. Whether emotions which increase colonic activity will also increase the likelihood of reflex anal dilatation is unknown but theoretically possible - so the fear of examination or the fear of the remembered pain of buggery may increase the chance of seeing reflex anal dilatation.

What is vital is that we avoid suppressing research or information which might help to clarify the issues even if this means that some of our widely held opinions may be proved wrong. No one should be compromised by acting according to the current state of knowledge provided they are flexible when new data arrive.

\section{References}

1 Clayden GS. Anal appearances and child sex abuse. Lancet 1987;i:620-1.

2 Clayden GS. Reflex anal dilatation associated with severe chronic constipation in childhood. Arch Dis Child 1988;63: 832-6.

\section{Reflex anal dilatation and sexual abuse}

Sir,

Dr Clayden's article highlights the serious problem facing all paediatricians. ${ }^{1}$ In a similar clinic in Newcastle upon Tyne, over six years, large numbers of severely constipated children have been treated with considerable success. Anal laxness has been observed in some children with heavily loaded rectums. Gross persistent dilatation has only been noted in five. Like Dr Clayden, I have 'satisfied myself' that all the children I see do not appear significantly disturbed and function well once treatment begins. A formal sexual abuse inquiry has never been initiated from this clinic.

Between January 1987 and July 1988, however, I have also been responsible for the evaluation of over 200 children referred from outside agencies, because of clear disclosures, or strong suspicions, of sexual abuse. At least $10 \%$ of these have had a background of successful paediatric involvement for a variety of chronic problems, including vulvovaginitis, constipation, soiling, or wetting.

Examples include a boy attending for severe chronic constipation and soiling over three years. This boy disclosed abuse within a week of respite fostering (introduced for non-relevant factors). He had not previously displayed significant disturbance apart from the constipation. What is more, this serious symptom, which had required two anaesthetic manipulations and three admissions to hospital in the past, disappeared within three weeks of the disclosure. A girl referred by her general practitioner for venereal disease had been discharged eight weeks earlier after successful management of acquired megacolon over two and a half years in a paediatric clinic.

When a child discloses abuse, anal dilatation is undeniably strongly corroborative of that abuse. It would be foolish to ignore the same sign when a child presents with a recognised symptom of abuse. The presence of hard stool at the internal sphincter is not an uncommon finding, anal dilatation is. The fact that the sign is variable, depending on the severity of withholding, may simply reflect the variability of stool withholding depending on whether the abusive episode is recent or not.

Dr Clayden appears convinced he has established a system which enables him to exclude abuse in his patients. I have examined over 300 childhood victims of sexual abuse and have listened to their life stories. Despite this experience, I do not feel I have developed the skills to detect abuse within the confines and resources of the outpatient constipation clinic.

When sexual abuse is being manifest in a silent child by years of severe constipation and soiling, it is unrealistic to expect that silence to be broken in the process of medical intervention. Nor should we expect to see unequivocal behavioural evidence, enabling us to investigate with impunity. It seems to me quite extraordinary that formal sexual abuse inquiry should continually appear to stem from agencies outwith the paediatric department, when serious symptomatology is daily managed within.

\section{Reference}

1 Clayden GS. Reflex and dilatation associated with severe chronic constipation in children. Arch Dis Child 1988;63:832-6.

$$
\begin{array}{r}
\text { C DE SAN LAZARo } \\
\text { Children's Clinic, } \\
\text { Royal Victoria Infirmary, } \\
\text { Newcastle upon Tyne NE1 4LP }
\end{array}
$$

Sir,

We read the article by Dr Clayden on reflex anal dilatation in severe constipation with interest. We note that of his 129 constipated children, $20(15 \%)$ had 'visibly relaxed anal sphincters'. ${ }^{1}$ Most of the children had received invasive treatment (anal dilatation, enemas, or suppositories) and all were tertiary referrals.

In view of the current debate on anal dilatation in constipation and sexual abuse we have assessed the anal appearances in children presenting to this hospital with constipation. Infants were examined supine and children in the left lateral position, with the minimum separation of the buttocks sufficient to visualise the anus. We recorded the presence or absence of anal dilatation, specifying whether this was of the external sphincter alone (partial) or of both the external and internal sphincters (full), and whether this occurred immediately or by 30 and 60 seconds. We then recorded the level of dilatation after gentle stretching of the buttocks. The full dilatation described here corresponds to the definition of reflex anal dilatation given in the report of the Cleveland inquiry. ${ }^{2}$

Over the last four months we have seen 72 constipated children with mild to moderate constipation, with or without soiling. All were primary general practitioner referrals and only one had received invasive treatment (suppositories). There were 39 boys and 33 girls aged 9 weeks to 12 years. Of these children, four showed partial 